\title{
Cross Section Dependence of Event Rates at Neutrino Telescopes
}

\author{
S. Hussain, ${ }^{1}$ D. Marfatia, ${ }^{2}$ D. W. McKay, ${ }^{2}$ and D. Seckel ${ }^{1}$ \\ ${ }^{1}$ Department of Physics and Astronomy, University of Delaware, Newark, Delaware 19716 USA \\ ${ }^{2}$ Department of Physics and Astronomy, University of Kansas, Lawrence, Kansas 66045 USA
}

(Received 3 July 2006; published 17 October 2006)

\begin{abstract}
We examine the dependence of event rates at neutrino telescopes on the neutrino-nucleon cross section for neutrinos with energy above $1 \mathrm{PeV}$, and contrast the results with those for cosmic ray experiments. Scaling of the standard model cross sections leaves the rate of upward events essentially unchanged. Details, such as detector depth and cross section inelasticity, can influence rates. Numerical estimates of upward shower, muon, and tau event rates in the IceCube detector confirm these results.
\end{abstract}

DOI: 10.1103/PhysRevLett.97.161101

Introduction.-Neutrinos with energy above $\mathcal{O}\left(10^{5}\right) \mathrm{GeV}$ have not been detected. They are sought after in both neutrino telescopes and cosmic ray experiments for two reasons. One, they may point back to (new) sources at cosmological distances and reveal the structure of their internal engines. Two, they enable the study of neutrino interactions at energies far beyond that achievable at colliders. The latter is especially interesting from a particle physics point of view because neutrinos only interact weakly according to the standard model (SM). Any new physics that affects how particles interact is therefore more likely to be detected in the neutrino sector. However, practical issues hamper the extraction of such information. Knowledge of astrophysical sources and fundamental particle interactions must be built up simultaneously, since we are largely ignorant of both in the energy regime of interest, and knowledge of one is dependent on knowledge of the other.

Currently, the situation is further complicated by confusion. Cosmic ray experiments detect upcoming leptons produced in the Earth by charged current interactions of "Earth-skimming" neutrinos. The effective area for detecting these upward leptons is a narrow projection of the actual detector surface area. The cross section dependence for the rate of such events has been derived in Refs. [1-3]. Subsequently, these results have been employed to assess the potential of neutrino telescopes [4,5], where they are inapplicable. Our motivation is to elucidate clearly (via qualitative reasoning, analytical calculation, and numerical analysis) how event rates at neutrino telescopes depend on the cross section and to show why the event rates in cosmic ray experiments have a different cross section dependence. We emphasize the role of the different cross sections and inelasticity in neutrino propagation and in the generation of upward shower and upward lepton events in providing probes for new physics. We begin by using simple models to determine the general dependence of the event rates on the cross sections that govern propagation and detection, and apply these concepts to different experimental configurations. We then reproduce our main results analytically. Finally, we perform numerical calculations of the
PACS numbers: 95.55.Vj, 13.15.+g

dependence of rates on cross section for the IceCube experiment [6], which confirm the conclusions of the simplified models.

Qualitative, geometric arguments. - Event rates are given by $\Gamma\left(E_{\nu}\right)=\frac{d \gamma}{d E_{\nu}}=\int d \Omega A_{e} \frac{d \phi}{d \Omega}$, where $A_{e}$ is the effective area for detection, and we use a diffuse, isotropic flux for $\frac{d \phi}{d \Omega}$. We assume that the dimensions of the detection volume are small compared to the length scale for neutrinos to be absorbed. We then approximate $A_{e}=n \sigma_{i} V_{e}$, where $V_{e}$ is the effective volume over which an interaction may be detected. $V_{e}$ can be expressed as $V_{e}=A_{p} \ell$, where $A_{p}$ is the area of the detector projected against the neutrino direction, and $\ell$ is the portion of the neutrino path to which the detector is sensitive; $n$ is the nucleon density within $V_{e}$; and $\sigma_{i}$ is the total (t), charged current (c) or neutral current (n) cross section corresponding to the process which makes the observable events. For shower detection, $\sigma_{i}=\sigma_{t}=$ $\sigma_{c}+\sigma_{n}$ as both charged and neutral current interactions produce showers, whereas for charged leptons $\sigma_{i}=\sigma_{c}$. Introducing the interaction length $\lambda_{i}=1 /\left(n \sigma_{i}\right)$, the effective area becomes $A_{e}=A_{p} \ell / \lambda_{i}=A_{p} \ell n \sigma_{i}$.

We first consider showers from downward neutrinos. Since showers are contained, $\ell$ is given by the detector scale $s$, so $A_{e}=A_{p} s / \lambda_{t}$. Typically, the detector is at a shallow depth $d$ compared to the absorption length for neutrinos, so one may integrate $d \Omega$ over the whole sky. The event rate for downward showers is

$$
\Gamma_{d, \mathrm{sh}}=2 \pi A_{p} \frac{d \phi}{d \Omega} \frac{s}{\lambda_{t}} \sim \sigma_{t},
$$

which increases with $\sigma_{t}$. The second case is the production of downward leptons. The effective area is given by $A_{e}=$ $A_{p} \ell / \lambda_{c}$. Here, $\ell$ is determined by the lepton stopping $(d E / d x)$ or decay, by the chord length to the surface, and by the detector size. We define leptons as detectable until they have lost $1 / e$ of their initial energy, after which they would be difficult to distinguish from more numerous lower energy leptons. This is a simple criterion, which can only be refined with a full experimental Monte Carlo simulation. Taking $d E / d x=-\gamma-\beta E$, the stopping range for lepton $j$ is $1 / \beta_{j}$ and $\gamma$ characterizes the ioniza- 
tion process. The decay length is $l_{j}=c \tau_{j} E / m_{j}$, and we denote the path length from the detector to the surface by $l(\theta)$. Qualitatively, $\ell=s / 2+\min \left(1 / \beta_{j}, l_{j}, l(\theta)\right)$, and the event rate is approximately

$$
\Gamma_{d, \text { lep }} \simeq \pi A_{p} \frac{d \phi}{d \Omega} \frac{s}{\lambda_{c}} \sim \sigma_{c},
$$

which scales as $\sigma_{c}$. For simplicity, we have suppressed the dependence on the depth of the detector.

Next, we turn to upward events and penetration through the Earth. We introduce the concept of neutrino attenuation length, $\lambda_{a}$ and attenuation cross section $\sigma_{a}=\sum_{i} \sigma_{i} y_{i}$, which is weighted by inelasticity. For neutral currents the inelasticity in the energy range under consideration, $\langle y\rangle \simeq$ $0.2-0.25$ [7], accounts for down scattering. For $\nu_{e}$ and $\nu_{\mu}$ charged current interactions, the charged leptons stop, so $y=1$ is appropriate. For $\nu_{\tau}$, the interplay between $\tau$ energy loss and decay gives an effective inelasticity in regeneration which increases with energy from about 0.6 to 1 [8]. For the SM at $10 \mathrm{PeV}, \sigma_{a} \simeq(0.77,0.77,0.5) \sigma_{t}$ for $\left(\nu_{e}, \nu_{\mu}, \nu_{\tau}\right)$

It simplifies the discussion to consider the case of a shallow detector near the surface for which $d=0$. The length of the chord traversed by an upward neutrino is $l=$ $2 R \sin \theta$, where $R$ is the radius of Earth and $\theta$ is the entry angle measured from the horizon. The attenuation length $\lambda_{a}$ limits the maximum chord length, so that the solid angle over which neutrinos are accepted scales as $\Omega=$ $2 \pi \sin \theta=\pi \lambda_{a} / R$. Using $A_{e}=A_{p} s / \lambda_{t}$, the rate for upward shower events is

$$
\Gamma_{u, \mathrm{sh}}=\pi A_{p} \frac{d \phi}{d \Omega} \frac{s}{R} \frac{\lambda_{a}}{\lambda_{t}} \sim \frac{\sigma_{t}}{\sigma_{a}} .
$$

Although the event rate will depend on details of $\sigma_{a}$ and $\sigma_{t}$, to the extent that all cross sections scale in a similar manner, the upward shower rate is independent of new physics. This is a consequence of the competition between two opposing effects: as the cross sections increase, the number of neutrinos entering the detector from below decreases with increased attenuation, but the probability for those neutrinos to interact increases.

The fourth case is upward leptons. We use $A_{e}=$ $A_{p} \ell / \lambda_{c}$. Folding in the reduced solid angle,

$$
\Gamma_{u, \text { lep }}=\pi A_{p} \frac{d \phi}{d \Omega} \frac{\ell}{R} \frac{\lambda_{a}}{\lambda_{c}} \sim \frac{\sigma_{c}}{\sigma_{a}} .
$$

If neutral current and charged current cross sections change proportionally, then the upward lepton rate is independent of cross section. However, if the new physics only adds to the shower rate, then the rate decreases due to the decrease in acceptance without a compensatory increase in the charged current interaction probability.

Since it was one of our motivating concerns, we examine the rate for cosmic ray air shower experiments to detect upward going leptons. In this case the detection criterion is that the leptons pass through a horizontal planar area $A_{0} \sim$ $s^{2}$ corresponding to the footprint of the cosmic ray experi- ment, and the projected area is $A_{p}=A_{0} \sin \theta$. Since $l=$ $2 R \sin \theta<\lambda_{a}, \sin \theta<\lambda_{a} /(2 R)$, so the projected area decreases with an additional factor of $1 / \sigma_{a}$. Integrating over solid angle,

$$
\Gamma_{\text {plane }} \simeq \frac{\pi}{2} A_{0} \frac{d \phi}{d \Omega} \frac{\ell}{R} \frac{\lambda_{a}}{R} \frac{\lambda_{a}}{\lambda_{c}} \sim \frac{\sigma_{c}}{\sigma_{a}^{2}} .
$$

We thus come to the conclusion of Ref. [1] that the event rate for upward leptons scales as $1 / \sigma_{a}^{2}$, where one factor of $\sigma_{a}$ comes from geometry, and one factor from $\sigma_{c} / \sigma_{a}$ assuming that the new physics does not increase the charged lepton production. However, we also see that this result does not apply to an in-ice detector such as IceCube, where the event rate for shower and charged lepton detection scales as $\sigma_{t} / \sigma_{a}$ and $\sigma_{c} / \sigma_{a}$, respectively.

The essential point of the above analysis for upward leptons is as follows. Suppose a detector can be described as a box with height $h$ and side $s$. The horizontal surface area is $A_{0}=s^{2}$ and the effective geometric area for incident direction $\theta$ is $A_{p}=s h \cos \theta+A_{0} \sin \theta \simeq s(h+s \theta)$. A detector like IceCube has $h \simeq s$ and $A_{p} \simeq A_{0}$, independent of $\theta$. Cosmic ray detectors have a planar geometry for which $h \ll s \theta$ and hence $A_{p} \simeq A_{0} \theta$. This additional factor of $\theta$ yields the extra factor of $1 / \sigma_{a}$ in Eq. (5).

Analytic model. - The probability that an upcoming neutrino propagates a distance $x$ along a chord is $P(x)=$ $e^{-x / \lambda_{a}}$, and the probability that it interacts and produces a detectable signature $i$ in the interval $d x$ is $d x / \lambda_{i}$. The rate of interactions is $\Gamma=A_{p} \frac{d \phi}{d \Omega} \int_{\ell}^{2 R} 2 \pi \frac{d l}{2 R} \int_{l-\ell}^{l} e^{-x / \lambda_{a}} \frac{d x}{\lambda_{i}}$, which on integration gives $\Gamma=\pi A_{p} \frac{d \phi}{d \Omega} \frac{\lambda_{a}^{2}}{R \lambda_{i}}\left(1-e^{-\ell / \lambda_{a}}\right) \times$ $\left(1-e^{-(2 R-\ell) / \lambda_{a}}\right)$. In the limit that $2 R \gg \lambda_{a} \gg \ell, \Gamma \simeq$ $\pi A_{p} \frac{d \phi}{d \Omega} \frac{\ell}{R} \frac{\lambda_{a}}{\lambda_{i}}$, which agrees with Eqs. (3) and (4).

For a surface detector, an additional factor of $\sin \theta=$ $l /(2 R)$ must be included in the $d l$ integral to project out the normal component of the lepton flux emerging from the Earth. For $2 R \gg \lambda_{a} \gg \ell$, we recover Eq. (5).

Numerical results. - So far, we have discussed neutrino event rates in various settings, but with several simplifying assumptions. We have used a shallow detector, a constant density Earth model, and have simplified the treatment of regenerated and down scattered neutrinos. To test our conclusions, we numerically estimate the event rates for upward showers and leptons produced by an isotropic total flux of neutrinos and antineutrinos $\frac{d \phi}{d \Omega}=6 \times$ $10^{-8}\left(E_{\nu} / \mathrm{GeV}\right)^{-2}\left(\mathrm{~cm}^{2} \text {.s.sr.GeV }\right)^{-1}$ [9] with an assumed flavor ratio at Earth of 1:1:1. The initial neutrino energies extend from $10^{6}$ to $10^{12} \mathrm{GeV}$. This model, used in Refs. [4,5], is for illustration only. A well-motivated alternative model produces a considerably larger flux relevant to IceCube [10].

We numerically solve the coupled Boltzmann equations [11] for three neutrino species and $\tau$ leptons, using a realistic Earth model to determine the flux of neutrinos at the detector. We include down scattering, $d E / d x$ for $\tau$ 's, and neutrinos from $\tau$ decay. For numerical integration, we 
divide the flux into 120 logarithmic energy bins. We solve for the flux in each of 41 angular bins, logarithmically spanning a range of $\theta$ from $\sim 0.1$ to $90^{\circ}$. The angular binning allows us to focus on narrowing angular ranges near the horizon as the cross sections increase.

Event rates are determined using ice as the detector medium for both showers and propagating charged leptons. We do not attempt to distinguish different event topologies, e.g., double bang, lollipop. Rather, we keep the simple criteria of the previous sections, i.e., that all showers are detected and that leptons have a practical range $\ell=s / 2+$ $\min \left(1 / \beta_{j}, l_{j}\right)$. We use $A_{p}=1.4 \mathrm{~km}^{2}$ and $V_{e}=2.25 \mathrm{~km}^{3}$, IceCube values [5] appropriate to the middle of the energy range under consideration, and infer $s=1.6 \mathrm{~km}$. We use $\beta_{\mu}=3.9 \times 10^{-6} \mathrm{~cm}^{-1}$ and $\beta_{\tau}=0.78 \times 10^{-6} \mathrm{~cm}^{-1}$, average values for ice in the same energy range.

We integrate across angles and present rates in logarithmic energy bins from $E_{\nu}=10^{6.5}-10^{9} \mathrm{GeV}$. The $\Delta \log _{10} E_{\nu}=0.5$ bin width is motivated by the eventual need to separate a smoothly changing flux and SM cross section from a hypothetical new physics cross section which may "turn on" over approximately a decade in energy. Our energy bins are for neutrino energy at the detector. A given bin may include down scattered neutrinos, or neutrinos produced in propagation by $\tau$ decay.

We parameterize physics models by three numbers $\alpha_{c}$, $\alpha_{n}$, and $\alpha_{n}^{\prime}$. The first two reflect the strength of charged and neutral current reactions, $\sigma_{i}=\alpha_{i} \sigma_{t}^{\mathrm{SM}}$, normalized to the SM total cross section. For our calculations, we take the inelasticity $d \sigma_{i} / d y$ to be the same as in the SM, and assume that the $\alpha_{i}$ are independent of energy. For $\alpha_{n}^{\prime}$, we define a new neutral current process also normalized to $\sigma_{t}^{\mathrm{SM}}$, but which is completely inelastic. Thus, the SM would be described by $\left(\alpha_{c}, \alpha_{n}, \alpha_{n}^{\prime}\right)=\left(r_{c}, r_{n}, 0\right)$, where $r_{i}=\sigma_{i}^{\mathrm{SM}} / \sigma_{t}^{\mathrm{SM}}$.

In Fig. 1, we show the expected number of IceCube events per year as a function of $\alpha$ for case I: a model with $\left(\alpha_{c}, \alpha_{n}, \alpha_{n}^{\prime}\right)=\left(\alpha r_{c}, \alpha r_{n}, 0\right)$, such as might occur if QCD saturation effects alter the growth predicted for neutrino-nucleon cross sections [12]. The upper panel shows shower rates in the five energy bins as a function of cross section for the nominal IceCube depth of $1.9 \mathrm{~km}$. For moderate values of $\alpha$, the rates are essentially independent of cross section, confirming Eq. (3). For low values of $\alpha$, the rates decrease because the Earth is no longer opaque, and at some point there is no compensating increase in flux to account for the reduced cross section. For large values of $\alpha$, the rates decrease because the horizontal distance to the surface, $\sqrt{2 d R}$, becomes comparable to the attenuation length $\lambda_{a}$. To confirm this, we redid the calculations with a shallow detector $(d=0)$. The results are consistent with a flat event rate, even for large $\alpha$. The slight increase in rates at large $\alpha$ is due to a decreased Earth density (and increase in $\lambda_{a}$ ) sampled by the neutrinos as their paths are constrained to lie closer to the surface. Since the rates are independent of cross section, the integrated rate in a bin drops as $1 / E_{\nu}$ for an $E_{\nu}^{-2}$ spectrum.

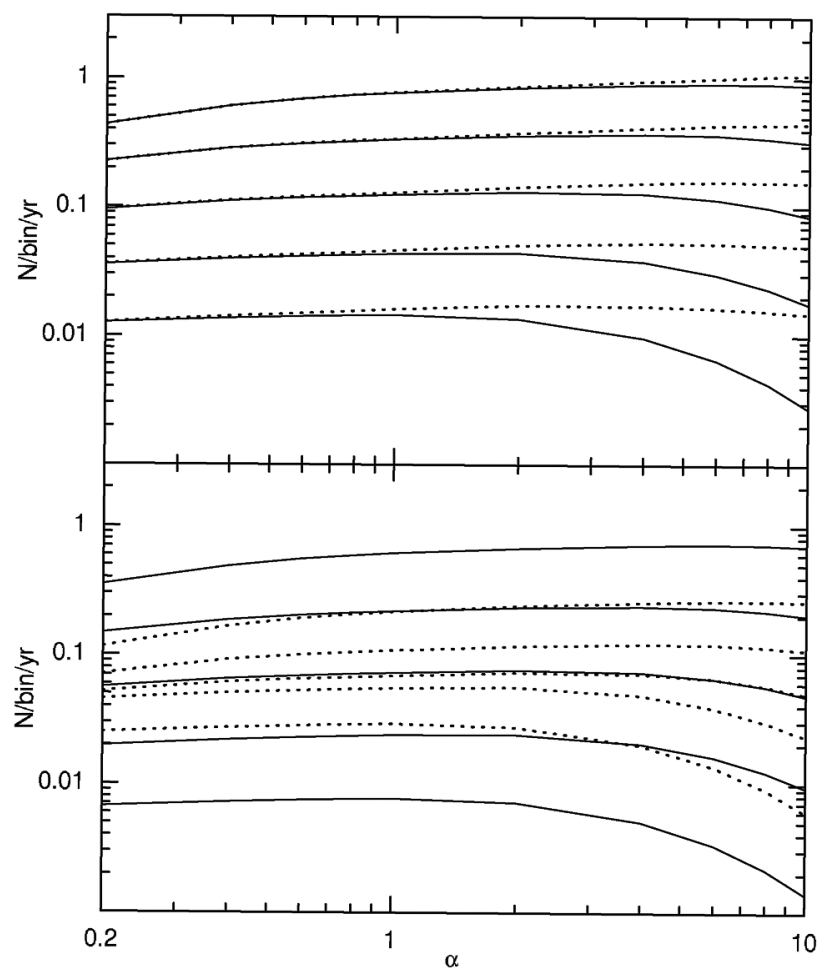

FIG. 1. Expected event rates at IceCube for $10^{6.5}<E_{\nu}<$ $10^{9} \mathrm{GeV}$ (in five bins of width $\Delta \log _{10} E_{\nu}=0.5$ ) for variation in strength of SM cross sections. Top: showers, solid line$1.9 \mathrm{~km}$ deep detector, dotted line-shallow detector. Bottom: leptons, solid line $-\mu$, dotted line $-\tau$. In each case, the uppermost curve corresponds to the lowest energy bin. We have adopted the flux of Ref. [9].

The lower panel shows similar results for $\mu$ and $\tau$ leptons, confirming Eq. (4). The main feature to note is that at low energy, the $\tau$ rates are low since $\tau$ decay limits $\ell$ to the detector size, while at high energies $\tau$ dominates since decays are delayed and $\beta_{\tau}<\beta_{\mu}$. Different assumptions about event identification (e.g., requiring double bang for $\tau$ ) would lead to different relative rates for $\mu$ and $\tau$. Integrating our results above $1 \mathrm{PeV}$, we find an expectation of $2.4,2.2$, and 0.9 events per year for upward shower, muons, and taus, respectively.

In Fig. 2, we show results for case II, a scenario where the SM cross sections are unaffected, but new interactions produce showers, i.e. $\left(\alpha_{c}, \alpha_{n}, \alpha_{n}^{\prime}\right)=\left(r_{c}, r_{n}, \alpha\right)$. An example would be a low scale gravity model [13], where microscopic black holes are produced by neutrino-nucleon interactions and then decay [3].

As for case I, Fig. 2 shows a shower rate that is essentially flat for moderate values of $\alpha$. The rate for leptons, however, falls inversely with $\alpha$ because $\sigma_{c}$ is fixed at the SM value while $\sigma_{a}$ is changed by new physics; see Eq. (4). The neutrino fluxes decrease because absorption increases, but this is not compensated by an increasing charged current interaction rate.

Figure 2 also shows results for a model with $\left(\alpha_{c}, \alpha_{n}, \alpha_{n}^{\prime}\right)=\left(r_{c}, r_{n}+\alpha, 0\right)$, i.e., where new neutral cur- 


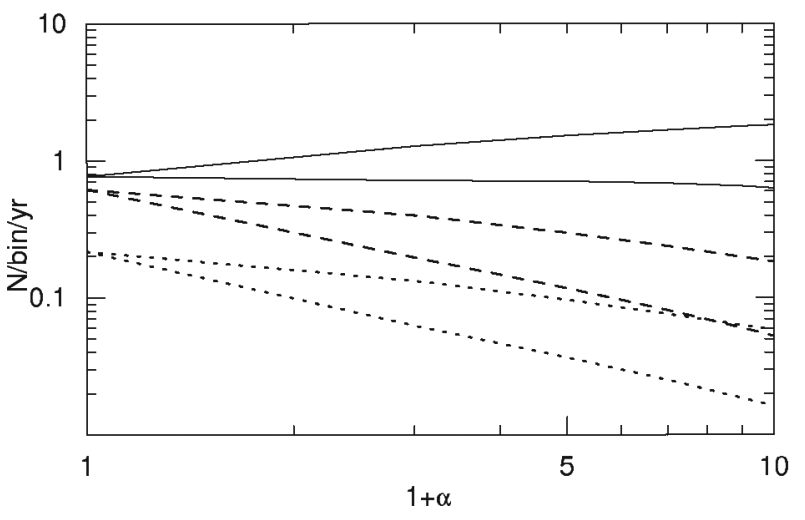

FIG. 2. Expected event rates for $10^{6.5}<E_{\nu}<10^{7} \mathrm{GeV}$ at IceCube for the case where new neutral current processes are added. For the "black hole" case (lower of each pair), the shower rate (solid line) is independent of cross section while the rates for leptons (dashed line $-\mu$, dotted line $-\tau$ ) fall with attenuation. For the case of "graviton exchange" (upper of each pair), reduced inelasticity and attenuation lead to higher rates.

rent interactions are added with inelasticity as in the SM, such as might occur due to graviton exchange. The difference between the two scenarios is due to differences in down scattering. In the first case, neutrinos are completely absorbed during propagation, while in the second they typically lose just $24 \%$ of their energy (at $10^{7} \mathrm{GeV}$ ) per interaction. Define the normalized attenuation cross section as $w_{a}=\sigma_{a} / \sigma_{t}^{\mathrm{SM}}$. Then the two scenarios give $w_{a}^{\prime} \simeq$ $0.77+\alpha$ and $w_{a} \simeq 0.77+0.24 \alpha$. For example, consider $\alpha=9$ on the right hand edge of Fig. 2. In this case, $w_{a}^{\prime} / w_{a} \simeq 3$, a ratio which is confirmed by the pairs of model curves in the figure.

Summary and discussion. - We have made simple scaling arguments to describe the expected detection rates for high energy neutrinos, with a focus on the IceCube experiment geometry. To validate our approach, we have performed numerical calculations which propagate a diffuse neutrino flux through the Earth.

Our first result is that the event rates for upward neutrinos in volume detectors are independent of the overall strength of the cross section. This conclusion is valid for over a decade change in the cross section normalization.

Our second conclusion is that the details matter. The event rates depend on a competition between neutrino attenuation and interaction lengths. The attenuation length depends on inelasticity $y$ as well as cross section, so models which alter $y$ from the SM value will also alter the scaling conclusion. In the extreme case of new totally inelastic showering interactions, the lepton rates are suppressed by a factor $\sim 1 /(1+\alpha)$ as can be seen from Fig. 2 .

Our third conclusion is that the definition of an "event" can lead to apples versus oranges comparisons. In our analysis, we required muons to retain most of their production energy, but did not require tau leptons to have an identifiable tag. On inclusion of tau event selection criteria and the implementation of tau energy losses as in Ref. [14], the expected SM rate for upward going taus with energy above $1 \mathrm{PeV}$ becomes 0.3 events/year. This is significantly smaller than the expectation in Refs. [4,5], whose extrapolation of the results of Ref. [14] above $10^{8} \mathrm{GeV}$ to values down to $10^{7} \mathrm{GeV}$ overestimates the tau flux at the detector by an order of magnitude.

The focus of this research is to understand the possibilities for inferring neutrino-nucleon cross sections when the neutrino flux is itself unknown. By taking a ratio of two separate measurements, a diffuse flux can be eliminated. If the ratio differs from the SM prediction, then something new is going on. The converse is not true-varying the SM cross sections, for example, would not affect the ratio of upward showers to upward muons. Several remedies suggest themselves. (a) Measure downward leptons; however, bundles of muons from cosmic ray air showers may provide an annoying background. (b) Measure the lepton rate near the horizon. The angular distribution is squeezed to the horizon as $\alpha$ increases. (c) Consider downward showers, which by our estimates form the majority of events. Perhaps the cleanest result would be a measurement of upward leptons with good angular resolution near the horizon, normalized to a total number of showers dominated by downward events.

We thank L. Anchordoqui, S. Barwick, H. Goldberg, D. Hooper, and $\mathrm{H}$. Reno for helpful communications. This research was supported by DOE Grant No. DE-FG0204ER41308, by NASA Grant No. NAG5-5390, by NSF Grant Nos. PHY-0544278, EPS-0236913, and OPP0338219, and by the State of Kansas through the KTEC.

[1] A. Kusenko and T. Weiler, Phys. Rev. Lett. 88, 161101 (2002); S. Palomares-Ruiz, A. Irimia, and T. Weiler, Phys. Rev. D 73, 083003 (2006).

[2] J. Feng et al., Phys. Rev. Lett. 88, 161102 (2002).

[3] L. Anchordoqui et al., Phys. Rev. D 65, 124027 (2002).

[4] L. A. Anchordoqui, J. L. Feng, and H. Goldberg, Phys. Rev. Lett. 96, 021101 (2006).

[5] L. Anchordoqui and F. Halzen, hep-ph/0510389.

[6] J. Ahrens et al., Astropart. Phys. 20, 507 (2004).

[7] R. Gandhi et al., Astropart. Phys. 5, 81 (1996).

[8] S. I. Dutta, M. H. Reno, and I. Sarcevic, Phys. Rev. D 62, 123001 (2000).

[9] E. Waxman and J. Bahcall, Phys. Rev. D 59, 023002 (1999).

[10] M. Ahlers et al., Phys. Rev. D 72, 023001 (2005).

[11] S. Hussain and D. McKay, Phys. Rev. D 69, 085004 (2004).

[12] L. Gribov, E. Levin, and M. Ryskin, Phys. Rep. 100, 1 (1983).

[13] N. Arkani-Hamed, S. Dimopoulos, and G. R. Dvali, Phys. Lett. B 429, 263 (1998); Phys. Rev. D 59, 086004 (1999); I. Antoniadis et al., Phys. Lett. B 436, 257 (1998).

[14] S. Dutta, Y. Huang, and H. Reno, Phys. Rev. D 72, 013005 (2005). 